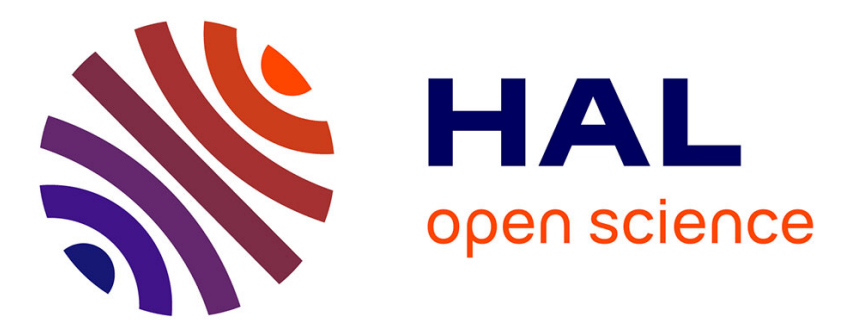

\title{
Hemodynamic simulations in the cerebral venous network: A study on the influence of different modeling assumptions
}

Vincent Chabannes, Mourad Ismail, Christophe Prud'Homme, Marcela Szopos

\section{- To cite this version:}

Vincent Chabannes, Mourad Ismail, Christophe Prud'Homme, Marcela Szopos. Hemodynamic simulations in the cerebral venous network: A study on the influence of different modeling assumptions. Journal of Coupled Systems and Multiscale Dynamics, 2015, 3 (1), pp.23-37. 10.1166/jcsmd.2015.1062 . hal-01109767v2

\author{
HAL Id: hal-01109767 \\ https://hal.science/hal-01109767v2
}

Submitted on 12 Mar 2015

HAL is a multi-disciplinary open access archive for the deposit and dissemination of scientific research documents, whether they are published or not. The documents may come from teaching and research institutions in France or abroad, or from public or private research centers.
L'archive ouverte pluridisciplinaire HAL, est destinée au dépôt et à la diffusion de documents scientifiques de niveau recherche, publiés ou non, émanant des établissements d'enseignement et de recherche français ou étrangers, des laboratoires publics ou privés.

$$
\text { Copyright }
$$




\title{
Hemodynamic simulations in the cerebral venous network: a study on the influence of different modeling assumptions
}

\author{
Vincent Chabannes * $\quad$ Mourad Ismail ${ }^{\dagger} \quad$ Christophe Prud'homme ${ }^{\ddagger} \quad$ Marcela Szopos $\S$
}

\begin{abstract}
Blood flow computations in complex geometries are of major interest in various cardio-vascular applications. However, deriving an appropriate computational model is still an open issue and a central question is how to incorporate and quantify uncertainties due to different modeling assumptions. The present work is intended as a first step in this direction, in the particular case of blood flow in the cerebral venous system. After a careful evaluation of the influence of the computational methodology, the study investigates the impact on the velocity field and the wall shear stress of three inflow boundary conditions, two strategies for treating the outflow boundary condition and two different viscosity models. The results demonstrate that the effect of setting the inflow boundary condition on the forces created by blood flow, is likely greater than for other modeling assumptions, the other important factor being the blood viscosity model, especially in wall shear stress computations. They suggest that improvements on the one hand on the mathematical and computational approach, and on the other hand on available data for their treatment are needed.
\end{abstract}

Keywords: Mathematical Modeling, Blood Flow Simulations, Cerebrovenous Hemodynamics, Finite Element Method, High Performance Computing.

MSC: 92C35 (Physiological flow), $76 Z 05$ (Physiological flows).

\section{Introduction}

In recent years, significant progress has been performed in blood flow simulations within geometrical models of vessels (see, for instance [12] and references therein for a sound monograph on this topic). However, several open issues still exist, in particular related to an accurate analysis of these complex multi-physics, multi-scale phenomena in complex geometries.

Moreover, although there is a vast literature assessing blood flow in the arterial network, to the best of our knowledge there are only a few studies exploring the venous part. There are several difficulties to tackle in modeling cerebral venous flow, such as: the asymmetric and considerably more various pattern of the venous network compared to the arterial one, the highly individual variations of the venous outflow [21], the compliant or even collapsible behavior of the venous wall etc. In addition, clinical measures provide only scarce and highly variable data in the cerebral venous tree, as illustrated by the values gathered hereafter: cross-sectional velocity, jugular vein: $(10-11) \times 10^{-2} \mathrm{~m} \cdot \mathrm{s}^{-1}$ [14], $(8.5-11.3) \times 10^{-2} \mathrm{~m} \cdot \mathrm{s}^{-1}$ [18], $(30-50) \times 10^{-2} \mathrm{~m} \cdot \mathrm{s}^{-1}$ [21]; cross-sectional velocity, superior sagittal sinus: $15 \times 10^{-2} \mathrm{~m} \cdot \mathrm{s}^{-1}$ [21]; venous mean cerebral blood flow $6.65 \pm 2.383 \times 10^{-6} \mathrm{~m}^{3} \cdot \mathrm{s}^{-1}$ for the right jugular vein, $2.683 \pm 2.5 \times 10^{-6} \mathrm{~m}^{3} \cdot \mathrm{s}^{-1}$ for the left jugular vein [23].

Nevertheless, some recent studies attempt to interpret blood flow in the cerebral venous network by means of a computational model, however mostly using a reduced order description in terms of geometric complexity. In this direction, we mention the recent cerebral venous one-dimensional flow model built on an anatomical geometry of the venous system proposed in Ref. [14], the closed-loop one-dimensional model for the human circulation, including a detailed description of the cerebral venous system developed in Ref. [17] and the 3D description of blood flow in rigid jugular veins coupled with a reduced one-dimensional model for major intracranial veins in Ref. [3].

In the present work, our aim is twofold: (i) provide a contribution to the modeling and simulation of blood flow in the venous compartment of the cerebral network, based on a full three-dimensional description of the geometry, reconstructed

\footnotetext{
*Laboratoire Jean Kuntzmann, Université Joseph Fourier Grenoble 1, BP53 38041 Grenoble Cedex 9, France

†Université Grenoble 1 / CNRS, Laboratoire Interdisciplinaire de Physique / UMR 5588. Grenoble, F-38041, France

‡Université de Strasbourg / CNRS, IRMA / UMR 7501. Strasbourg, F-67000, France

§Université de Strasbourg / CNRS, IRMA / UMR 7501. Strasbourg, F-67000, France. Corresponding author. Email: szopos@math.unistra.fr, Tel.: +33 (0)3.68.85.02.08, Fax: +33 (0)3 68850328 .
} 
from medical imaging [16]; (ii) investigate by means of a systematic numerical exploration the effects of different modeling assumptions on flow conditions.

To accomplish the above objectives, we consider the Navier-Stokes system of PDEs, valid in large and medium-sized cerebral veins [15], under the following standard assumptions: (i) the blood density is constant; (ii) the flow is incompressible and isothermal; (iii) a Newtonian or a Generalized Newtonian model is used for blood flow. More details are given in Section 2. Blood flow in rigid veins is then computed, on the basis of the methodology previously developed in [4] and using adequate values of the flow governing parameters and boundary conditions. The computational framework builds upon FEEL++, Finite Element Embedded Language in $C++$, [19], a flexible generic library which allows for arbitrary order continuous and discontinuous Galerkin methods in 1D, 2D and 3D, seamlessly in parallel. We describe the numerical and computational strategy, the sensitivity analysis framework and a detailed study of the influence of the numerical methods in Section 3. In Section 4 we present several numerical illustrations of large scale simulations of blood flow in complex geometries, and quantify the impact of variable modeling choices on the hemodynamic quantities of interest (velocity and wall shear stress). Finally, in Section 5 we discuss the significance of the obtained results and draw some perspectives, giving insight into the difficulties related to a deeper understanding of the clinical relevance of the solutions and into some potential solutions.

\section{A 3D model for blood flow in large cerebral veins}

The mathematical equations that govern blood flow dynamics in medium and large vessels are the homogeneous, incompressible, unsteady Navier-Stokes equations, which read in conservative form: find $(\mathbf{u}, p)$ such that

$$
\begin{array}{r}
\rho\left(\frac{\partial \mathbf{u}}{\partial t}+(\mathbf{u} \cdot \nabla) \mathbf{u}\right)-\operatorname{div}(-p \mathbf{I}+2 \mu \mathbf{D}(\mathbf{u}))=\mathbf{0} \\
\operatorname{div}(\mathbf{u})=0
\end{array}
$$

in $\Omega \times I$, supplemented with initial and boundary conditions. The set $\Omega \subset \mathbb{R}^{3}$ represents the spatial domain (the lumen of the vessels), $I=(0, T)$ is the time interval (one or several cardiac cycles), $\mathbf{u}$ and $p$ are the velocity and pressure of the fluid, $\mathbf{D}(\mathbf{u})=\frac{1}{2}\left(\nabla \mathbf{u}+\nabla \mathbf{u}^{T}\right)$ is the strain rate tensor and $\rho$ and $\mu$ are the density and the dynamic viscosity of the fluid, respectively.

The viscosity is taken either to be constant, when adopting a Newtonian constitutive model, or as a function of the shear rate:

$$
\dot{\gamma}=\sqrt{2 \operatorname{tr}\left(\mathbf{D}(\mathbf{u})^{2}\right)},
$$

when using a Generalized Newtonian model. In the present work, we focus on the comparison between the Newtonian model, and the Carreau and Carreau-Yasuda Generalized Newtonian constitutive models for blood [12, Chap. 6], that return the viscosity as a function of the shear rate by the following equation:

$$
\frac{\mu(\dot{\gamma})-\mu_{\infty}}{\mu_{0}-\mu_{\infty}}=\left(1+(\lambda \dot{\gamma})^{a}\right)^{\frac{n-1}{2}}
$$

where $\lambda$ is a time constant, $a$ and $n$ are dimensionless parameters used to differentiate between the two models, and $\mu_{0}$ and $\mu_{\infty}$ are the viscosities at zero and infinite shear rate, respectively (see also 4.2).

We define the Cauchy stress tensor

$$
\boldsymbol{\sigma}(\mathbf{u}, p)=-p \mathbf{I}+2 \mu \mathbf{D}(\mathbf{u})
$$

and the wall shear stress $\tau$ as being the magnitude of the surface traction vector:

$$
\mathbf{T}(\mathbf{u}, p)=\boldsymbol{\sigma}(\mathbf{u}, p) \mathbf{n}-((\boldsymbol{\sigma}(\mathbf{u}, p) \mathbf{n}) \cdot \mathbf{n}) \mathbf{n},
$$

where $\mathbf{n}$ indicates the outward normal to $\partial \Omega$.

Furthermore, in order to prescribe the initial status of the fluid velocity, we impose:

$$
\mathbf{u}(t=0, \mathbf{x})=\mathbf{u}_{0}(\mathbf{x}), \text { for } \mathbf{x} \in \Omega,
$$

where $\mathbf{u}_{0}$ is a given quantity such that $\operatorname{div}\left(\mathbf{u}_{0}\right)=0$. 
To specify the boundary conditions, we denote by $\Gamma_{\text {wall }}$ the lateral boundary (the vessel wall) and by $\Gamma_{\text {in }} / \Gamma_{\text {out }}$ the upstream / downstream boundaries. At the inflow of the cerebrovenous network, blood comes from the microcirculation, modeled by a quasi-steady / steady Stokes flow, hence we impose a constant profile of small magnitude on $\Gamma_{\text {in }}$. The vessel wall is considered to be rigid, hence $\mathbf{u}=\mathbf{0}$ on $\Gamma_{\text {wall, }}$, which is an acceptable hypothesis when studying intracranial arterial blood flow [22]. However, extracranial veins are less stiff than the intracranial ones [23], but as a first approximation, we also consider the jugulars as being rigid; the extension to the compliant case is postponed to future work. At the outflow, boundary conditions are prescribed either by using a traction-free condition largely employed in blood flow simulations:

$$
\boldsymbol{\sigma}(\mathbf{u}, p) \mathbf{n}=\mathbf{0} \quad \text { on } \quad \Gamma_{\text {out }},
$$

or by introducing the coupling with a three-element Windkessel model [12, Chap. 10], in order to take into account the downstream vasculature. In the latter case, the condition reads:

$$
\boldsymbol{\sigma}(\mathbf{u}, p) \mathbf{n}=-P_{l} \mathbf{n} \quad \text { on } \quad \Gamma_{\text {out }},
$$

where $P_{l}$ (the proximal pressure) is obtained by solving

$$
\left\{\begin{array}{c}
C_{d, l} \frac{d \pi_{l}}{d t}+\frac{\pi_{l}}{R_{d, l}}=Q_{l} \\
P_{l}=R_{p, l} Q_{l}+\pi_{l} .
\end{array}\right.
$$

for a given value of the flux on the outlet $Q_{l}=\int_{\Gamma_{\text {out }}} \mathbf{u} \cdot \mathbf{n} \mathrm{d} \mathbf{x}$. Further details supporting the present choices for the boundary conditions can be found in [15]. It should be noted that more involved models, as for instance a 3D - 1D coupled system, could be developed in the sequel, in order to better take into account the downstream, possibly compliant, vasculature.

\section{Numerical method and computational framework}

\subsection{Numerical Method}

We now turn to the discretization of the mathematical models presented in the previous section. We start with the spacetime discretization of the incompressible Navier-Stokes equations: we use a fully implicit time discretization using $\mathrm{BDF}_{\eta}$ scheme including for the Non-Newtonian models with non-linear solves handled by a Newton method. The equations (1) time discretized read

$$
\begin{aligned}
& \rho\left(\frac{\sum_{k=0}^{\eta} \alpha_{k} \mathbf{u}^{n+1-k}}{\Delta t}+\mathbf{u}^{n+1} \cdot \nabla \mathbf{u}^{n+1}\right) \\
&-\operatorname{div}\left(-p^{n+1} \mathbf{I}+2 \mu \mathbf{D}\left(\mathbf{u}^{n+1}\right)\right)=\mathbf{0}, \\
& \operatorname{div}\left(\mathbf{u}^{n+1}\right)=0 .
\end{aligned}
$$

where $\left(\alpha_{k}\right)_{k=0, \eta}$ are the $\mathrm{BDF}_{\eta}$ coefficients for the time derivative of $\mathbf{u}$ and the subscript $\eta$ refers to the order of the scheme. In the sequel, we adopt the $B D F_{1}(\eta=1)$ and $B D F_{2}(\eta=2)$ schemes for the time approximation. The spatial discretization is handled via a inf-sup stable finite element (Taylor-Hood) $\mathbb{P}_{2}-\mathbb{P}_{1}$, see e.g. [20]. The weak formulation associated to (10) reads: find $\left(\mathbf{u}_{h}, p_{h}\right)$ such that for all $\left(\mathbf{v}_{h}, q_{h}\right)$

$$
\begin{aligned}
\int_{\Omega} \rho\left(\frac{\mathbf{u}_{h}^{n+1} \cdot \mathbf{v}_{h}}{\Delta t}+\mathbf{u}_{h}^{n+1} \cdot \nabla \mathbf{u}_{h}^{n+1} \cdot \mathbf{v}_{h}\right) \mathrm{d} \mathbf{x} & \\
& +\int_{\Omega} 2 \mu \mathbf{D}\left(\mathbf{u}_{h}^{n+1}\right): \mathbf{D}\left(\mathbf{v}_{h}\right)+p_{h}^{n+1} \nabla \cdot \mathbf{v}_{h}+q_{h} \nabla \cdot \mathbf{u}^{n+1} \mathrm{~d} \mathbf{x} \\
& \quad+\int_{\partial \Omega}\left(-p_{h}^{n+1} \mathbf{n}+2 \mu \mathbf{D}\left(\mathbf{u}_{h}^{n+1}\right) \mathbf{n}\right) \cdot \mathbf{v}_{h} \mathrm{~d} \mathbf{x} \\
& =-\int_{\Omega} \frac{\sum_{k=1}^{\eta} \alpha_{k} \mathbf{u}^{n+1-k} \cdot \mathbf{v}_{h}}{\Delta t} \mathrm{~d} \mathbf{x}
\end{aligned}
$$

The boundary integral in (11) is handled through the boundary conditions on $\Gamma_{\mathrm{in}}, \Gamma_{\text {out }}$ and $\Gamma_{\text {wall }}$ presented in Section 2. 
From an algebraic point of view, at each non-linear iteration, the following classical saddle point system is inverted

$$
\left(\begin{array}{cc}
A & B^{T} \\
B & 0
\end{array}\right)\left(\begin{array}{l}
U \\
P
\end{array}\right)=\left(\begin{array}{l}
F \\
0
\end{array}\right)
$$

where $A$ corresponds to the velocity block and $B, B^{T}$ to the velocity/pressure coupling. This system is solved with GMRES using the SIMPLE preconditioner, see [20].

In the case of the 3D-0D implicit coupling, the following system is inverted

$$
\left(\begin{array}{ccc}
A & B^{T} & D \\
B & 0 & 0 \\
C & 0 & E
\end{array}\right)\left(\begin{array}{l}
U \\
P \\
\Lambda
\end{array}\right)=\left(\begin{array}{c}
F \\
0 \\
G
\end{array}\right)
$$

where $\Lambda$ is the vector $\left(\pi_{l}, P_{l}\right)$, the blocks $C$ and $D$ correspond to the coupling between velocity and the 0D variables and the blocks $E$ and $G$ the 0 D variables block and data respectively. This system is solved in two embedded steps: $(i)$ a block GAUSS-SEIDEL preconditioner is first applied to the 3D-0D blocks (velocity and pressure are aggregated in the 3D block) then (ii) the SIMPLE preconditioner is applied to the 3D block only, i.e. on the velocity/pressure saddle-point problem. This strategy needs however to be refined in further work as it is currently more expensive than the traction-free model (approximatively 5 Gauss-Seidel iterations are necessary to achieve convergence, hence about 5 times slower).

As to time discretization, the 0D time scheme has the same accuracy as the time discretization scheme used for the Navier-Stokes equations. Note that an explicit scheme is also available and has been compared with the implicit one described above, see Section 3.4 .5

\subsection{Computational framework}

The analysis hereafter used the Finite Element Embedded Library in C++ (Feel++). Feel++ allows to use a very wide range of Galerkin methods and advanced numerical methods such as domain decomposition methods including mortar and three fields methods, fictitious domain methods or certified reduced basis. The ingredients include a very expressive embedded language, seamless interpolation, mesh adaption and seamless parallelization. It has been used in various contexts including the development and/or numerical verification of (new) mathematical methods or the development of large multi-physics applications [4]. The range of users span from mechanical engineers in industry, physicists in complex fluids, computer scientists in biomedical applications to applied mathematicians thanks to the shared common mathematical embedded language hiding linear algebra and computer science complexities.

Feel++ provides a mathematical kernel for solving partial differential equation using arbitrary order Galerkin methods (FEM, SEM, CG, DG, CRB) in 1D, 2D, 3D and manifolds using simplices and hypercubes meshes [19]: (i) a polynomial library allowing for a wide range polynomial expansions including $H_{\text {div }}$ and $H_{\text {curl }}$ elements, (ii) a light interface to Boost.UBLAS, EIGEN3 and PETSC/SLEPC as well as a scalable in-house solution strategy (iii) a language for Galerkin methods starting with fundamental concepts such as function spaces, forms, operators, functionals and integrals, (iv) a framework that allows user codes to scale seamlessly from single core computation to thousands of cores and enables hybrid computing.

Feel++ takes advantage of the newest $\mathrm{C}++$ standard $(\mathrm{C}++11)$ such as type inference and the Boost $\mathrm{C}++\mathrm{Libraries}$ such as the Boost.PARAmeter, Boost.Fusion or Boost.MPL and many more. These language enhancements and libraries allow for very concise, robust and expressive $\mathrm{C}++$ codes. The current paper illustrates the capabilities of this framework in terms of modeling as well as high performance computing.

The analyses presented in the next sections were partly performed on SuperMUC at LRZ in Munich, Germany, which provides 155.000 cores and a peak performance of 3 Pflop $\cdot \mathrm{s}^{-1}$, the MesoCenters (Tier-2) in Strasbourg and Grenoble offering about $70 \mathrm{Tflop} \cdot \mathrm{s}^{-1}$ and $45 \mathrm{Tflop} \cdot \mathrm{s}^{-1}$ as peak performance respectively. The computations including post-processing analysis were distributed from 64 to 512 cores depending on the cost of the study. The simulations ran from 10 to $15 \mathrm{~h}$ and the post-processing from 5 to $20 \mathrm{~h}$.

\subsection{Sensitivity analysis setup}

The sensitivity analysis framework in the present paper stems from [10], where the author defines two metrics, one for the velocity and one for the wall shear stress in order not only to visually compare snapshots of solutions, but also to assess the differences between two solutions in a more quantitative manner. Both metrics require to define a computed reference 
solution (see Sec. 3.3.1) and then the computation of the time evolution of the average on the entire mesh of some specific quantities (see Sec. 3.3.2 and 3.3.3) which reflect pointwise differences between velocities and the wall shear stresses, respectively. This approach has in addition the advantage of assessing the time evolution of the these differences, whereas comparisons of snapshots are only showing spacial differences at some selected instants.

\subsubsection{Computed reference solution}

We consider the cerebrovenous network obtained from [16], see Figure 1, with 29 inlets with blood coming from the microcirculation and 2 outlets corresponding to the jugulars.

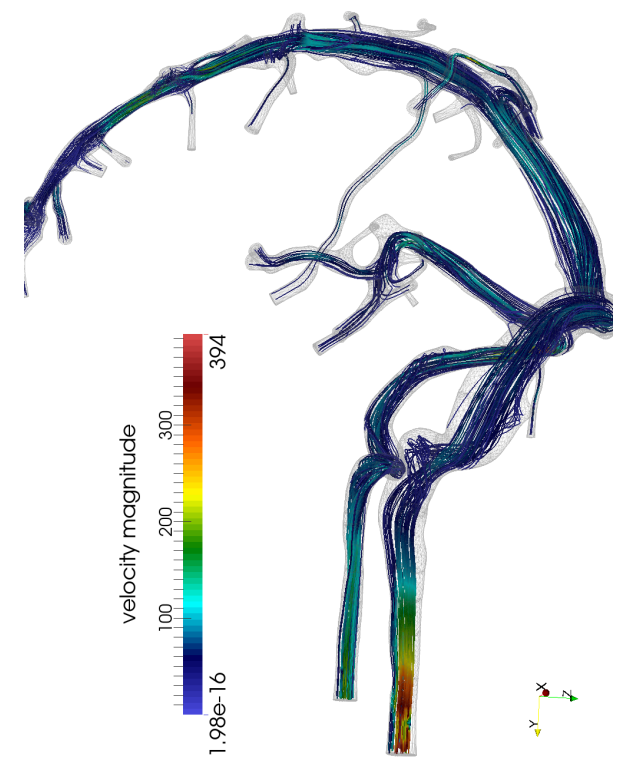

Figure 1: Visualization of the flow patterns: instantaneous streamlines, colored with velocity magnitude (in $\left.\mathrm{mm} \cdot \mathrm{s}^{-1}\right)$.

The computed reference solution for the sensitivity analysis is obtained as follows: (i) we consider blood to be a Newtonian fluid with viscosity $\mu=3.4815 \times 10^{-3} \mathrm{~Pa} \cdot \mathrm{s}$ and density $\rho=1.055 \times 10^{3} \mathrm{~kg} \cdot \mathrm{m}^{-3}$ modeled by the unsteady incompressible Navier-Stokes equations, with constant inflow velocity $v_{\text {in }}$ (physiological range) over $\Gamma_{\text {in }}$, rigid walls for $\Gamma_{\text {wall }}$, traction-free outflow on $\Gamma_{\text {out }}$; (ii) a fully implicit time discretization using a $\mathrm{BDF}_{2}$ scheme with a constant time step $\Delta t=10^{-3}$ s and the final time $T=1 \mathrm{~s} ;$ (iii) a stable Taylor-Hood spatial discretization $\mathbb{P}_{2}-\mathbb{P}_{1}$ using a fine grid accounting for about 10 millions degrees of freedom, see Table 1 for a list of meshes.

The reference solutions may vary with respect to the inflow velocity $v_{\text {in }}$ within the set $\left\{10 \times 10^{-3}, 20 \times 10^{-3}, 30 \times 10^{-3}\right\} \mathrm{m} \cdot \mathrm{s}$ Unless notified, the default value is $v_{\text {in }}=10 \times 10^{-3} \mathrm{~m} \cdot \mathrm{s}^{-1}$.

\subsubsection{Velocity metric}

This metric measures the difference between the computed solution $\left(\mathbf{u}_{C}\right)$ and the computed reference solution $\left(\mathbf{u}_{R}\right)$. To this end, [10] defines the spatial metric for each time step

$$
S_{\mathbf{u}_{R}}\left(\mathbf{u}_{C}\right)=1-\beta e^{-a}-\gamma e^{-m},
$$

where

$$
\begin{aligned}
a & =\frac{1}{\pi} \cos ^{-1}\left(\frac{\mathbf{u}_{R} \cdot \mathbf{u}_{C}}{\left\|\mathbf{u}_{C}\right\|\left\|\mathbf{u}_{C}\right\|}\right), \quad 0 \leq a \leq 1, \\
m & =\frac{\left\|\mathbf{u}_{R}-\mathbf{u}_{C}\right\|}{\left\|\mathbf{u}_{R}\right\|}, \quad 0 \leq m \quad \text { and } \quad \beta=\gamma=\frac{1}{2}
\end{aligned}
$$


The value $a$ corresponds to the scaled angle between the two vectors and the value $m$ to relative difference in their order of magnitude. The spatial average is then computed by:

$$
D_{\mathbf{u}}=\frac{1}{V} \int_{\Omega} S_{\mathbf{u}_{R}}\left(\mathbf{u}_{C}\right) \mathrm{d} \mathbf{x}, \quad \text { where } V=\int_{\Omega} 1 \mathrm{~d} \mathbf{x} .
$$

\subsubsection{Wall shear stress metric}

This metric measures the difference between the computed reference solution of the wall shear stress $\tau_{R}$ and another numerical solution of the wall shear stress $\tau_{C}$. To this end, [10] defines the spatial metric for each time step as

$$
T_{\tau_{R}}\left(\tau_{C}\right)=1-e^{-t}, t=\left|\frac{\tau_{R}-\tau_{C}}{\tau_{R}}\right|
$$

and its spatial average

$$
D_{\tau}=\frac{1}{S} \int_{\partial \Omega} T_{\tau_{R}}\left(\tau_{C}\right) \mathrm{d} \mathbf{x}, \text { where } S=\int_{\partial \Omega} 1 \mathrm{~d} \mathbf{x}
$$

\subsubsection{Metric computations}

We discuss here some details regarding the metric computations. Once the simulations for a given study are done, the analysis follows Algorithm 1 The function LoadVelocityAndWSS loads the parallel data containing the velocity $\mathbf{u}_{C}$ and wall shear stress $\tau_{C}$ associated to the time $t$ of a given simulation as well as the corresponding reference solution $\mathbf{u}_{R}$ and $\tau_{R}$. We next compute the metrics $D_{\mathbf{u}}$ and $D_{\tau}$ for each time step.

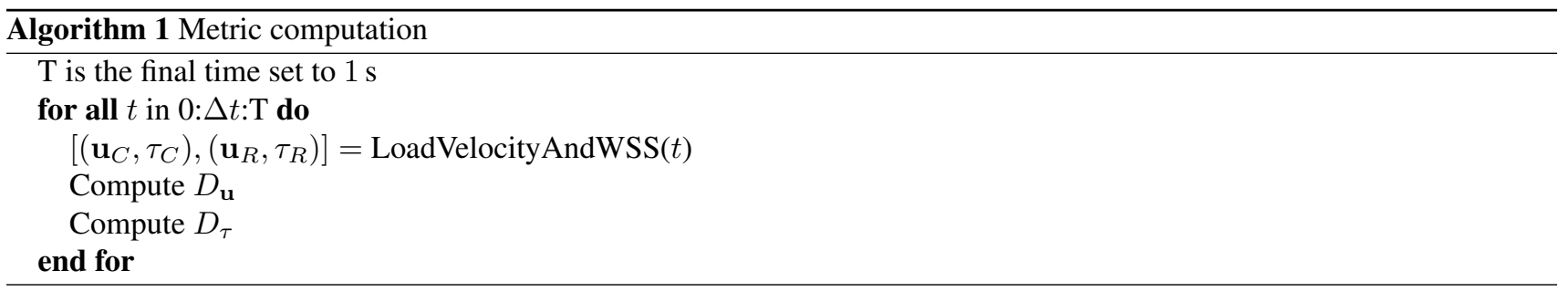

We first note that division by zero can occur in both metrics and it needs to be handled by adding safeguards in the integral computations to discard these cases. As to the volume and surface integrals, they are computed using quadratures that integrate exactly up to order 6 polynomials in 3D since the integrands are non-trivial expressions including the aforementioned safeguards. Thus Algorithm 1 requires very intensive parallel computation to obtain the metrics (the studies used from 64 to 512 cores and ranged from 1 Million to about 10 Millions degrees of freedom). In our case they were almost as expensive as some of the actual numerical simulations, see Section 3.2 .

\subsection{Influence of the numerical strategy}

Before investigating the impact of several modeling assumptions, our objective in the present section is to assess the impact of the numerical strategy in use. We ensure that our sensitivity analysis is performed in a controlled numerical environment within the open-source developed and validated software library Feel++, see section 3.2, and in [5, 6, 9, 8, 4]. Our numerical strategy is in line with [24] as we execute high resolution simulations and not normal resolution simulations: both the spacial and the temporal discretization methods are second-order schemes and a small time step $\left(\Delta t=10^{-3} \mathrm{~s}\right)$ was chosen, in order to adequately resolve the complex flow features. In the following sections, we apply the sensitivity analysis to various numerical choices namely space time discretization, handling of the non-linearity and 3D-0D coupling.

\subsubsection{Mesh convergence}

We start with the mesh convergence which has been done on three meshes M0, M1 and M 2 described in Table 1 applied to the computed reference solution described in section 3.3.1 with inlet velocity $v_{\text {in }}=10 \times 10^{-3} \mathrm{~m} \cdot \mathrm{s}^{-1}$ and the computed solution with inlet velocity $v_{\text {in }}=30 \times 10^{-3} \mathrm{~m} \cdot \mathrm{s}^{-1}$. The results are displayed in Figure 2. We expect that the sensitivity with respect to $v_{\text {in }}$ tends to the same profile as the mesh is refined. Indeed the results show that (i) the numerical simulations 
are quite sensitive to the inlet velocity value $v_{\text {in }}$; (ii) the coarser mesh $\mathrm{M} 0$ provides already good results when compared with finer meshes; and (iii) the curves for M1 and M2 are superimposed, showing that mesh independence is achieved with the second level of refinement. Similar results are obtained with $v_{\text {in }}=20 \times 10^{-3} \mathrm{~m} \cdot \mathrm{s}^{-1}$.

\begin{tabular}{rrrr}
\hline & $h$ & $N_{\text {elt }}$ & $N_{\text {dof }}$ \\
\hline M0 & 0.03 & 322013 & 1640236 \\
M1 & 0.02 & 985484 & 4717123 \\
M2 & 0.015 & 2008757 & 9171904 \\
\hline
\end{tabular}

Table 1: Mesh convergence tests; $h$ : characteristic element size, $N_{\text {elt }}:$ number of tetrahedra, $N_{\text {dof }}:$ number of degrees of freedom.

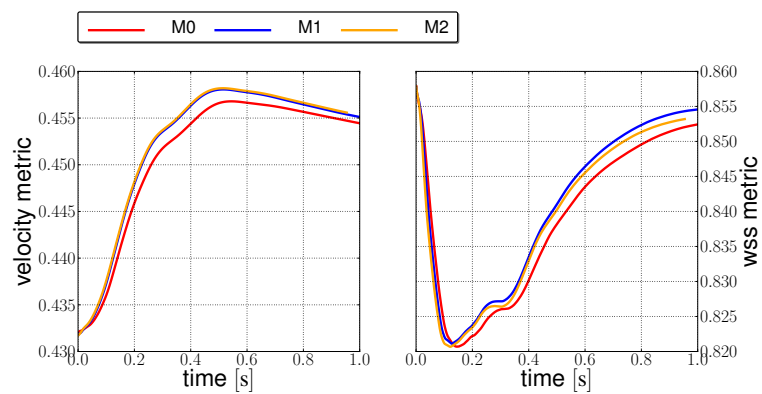

Figure 2: Sensitivity indices for the different grids $\mathrm{M} 0, \mathrm{M} 1$ and $\mathrm{M} 2$ with inlet velocity $v_{\mathrm{in}}=30 \times 10^{-3} \mathrm{~m} \cdot \mathrm{s}^{-1}$ and with respect to the reference solution $v_{\text {in }}=10 \times 10^{-3} \mathrm{~m} \cdot \mathrm{s}^{-1}$.

\subsubsection{Initial condition}

We look now at the choices for the initial solution: (i) fluid at rest, (ii) Stokes solution. The results are displayed in Figure 3 where the reference solution for each inlet velocity is the Navier-Stokes Newtonian model with initial solution at rest, i.e. $(\mathbf{u}=\mathbf{0}, p=0)$ and the same inlet velocity. The sensitivity to the initial condition choice decreases significantly over $1 \mathrm{~s}$ and can be discarded afterwards.

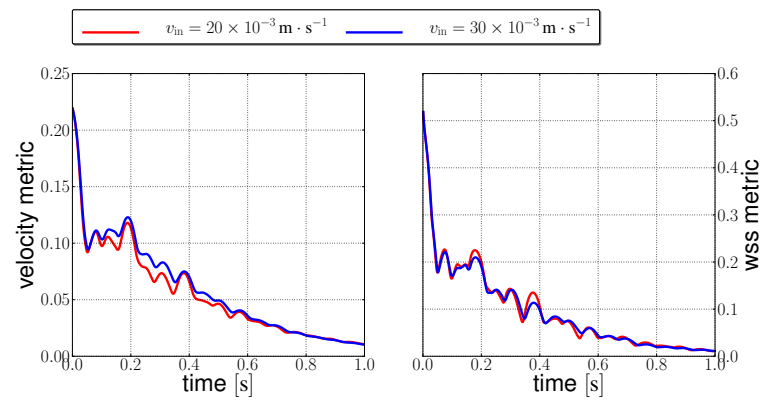

Figure 3: Initial condition sensitivity.

\subsubsection{Time-discretization order}

We are now interested in the effect of the time step scheme for Navier-Stokes with $v_{\text {in }}=30 \times 10^{-3} \mathrm{~m} \cdot \mathrm{s}^{-1}$. The reference solution is computed with $\mathrm{BDF}_{1}$ with the same time step $\Delta t=10^{-3} \mathrm{~s}$. A comparaison with the second order scheme $B D F_{2}$ is displayed in Figure 4 and shows that the results are relatively insensitive to the time discretization order. 

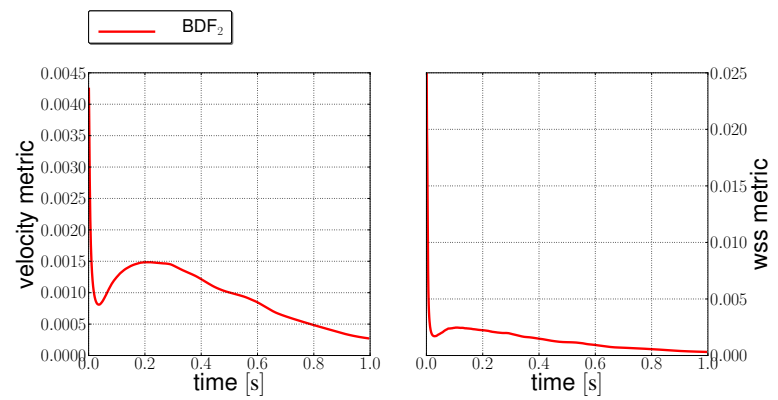

Figure 4: $B D F_{1}$ vs. $B D F_{2}, \Delta t=10^{-3} \mathrm{~s}$, inlet velocity $v_{\text {in }}=30 \times 10^{-3} \mathrm{~m} \cdot \mathrm{s}^{-1}$.

\subsubsection{Fully implicit versus semi-implicit}

We consider here the sensitivity to the choice between a fully implicit and semi implicit (Oseen) scheme to handle the Navier-Stokes equations. The analysis was conducted with a reference computed solution with the highest inlet velocity $v_{\text {in }}=30 \times 10^{-3} \mathrm{~m} \cdot \mathrm{s}^{-1}$. The results are displayed in Figure 5 and from the very small values for both the velocity and the wall shear stress metrics we infer that that in this case the computed solution is insensitive to the scheme choice.
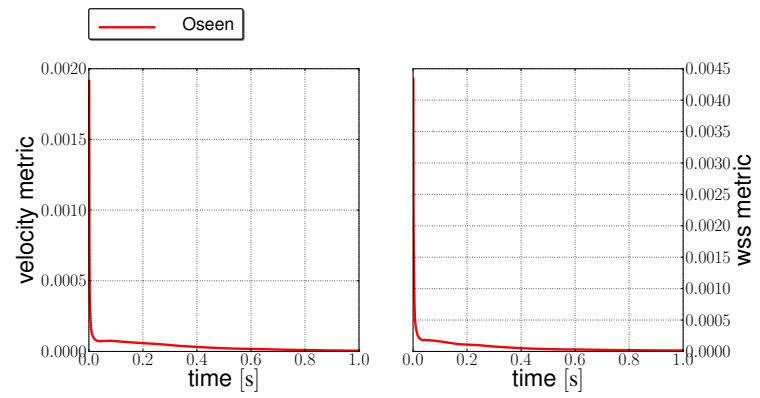

Figure 5: Fully implicit vs. Semi-implicit (Oseen) scheme, $\Delta t=10^{-3} \mathrm{~s}$, inlet velocity $v_{\text {in }}=30 \times 10^{-3} \mathrm{~m} \cdot \mathrm{s}^{-1}$.

\subsubsection{Implicit versus explicit 3D-0D coupling}

Finally we analyze the sensitivity to the implicit versus explicit treatment of the 3D-0D coupling. It was conducted with a reference computed solution with the highest inlet velocity $v_{\text {in }}=30 \times 10^{-3} \mathrm{~m} \cdot \mathrm{s}^{-1}$. The results are displayed in Figure 6 and they show that they are sensitive to this choice, the indices decrease over $1 \mathrm{~s}$ and seem to stagnate towards the end of the simulation. It should be noted that the explicit scheme is stable in our case, thanks probably to the small time-step, but this is not always the case, see [2].
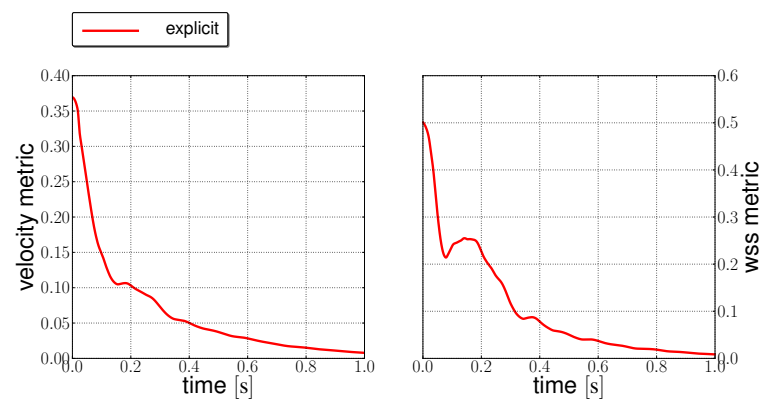

Figure 6: 3D-0D coupling, implicit vs. explicit scheme, inlet velocity $v_{\text {in }}=30 \times 10^{-3} \mathrm{~m} \cdot \mathrm{s}^{-1}$. 


\section{Application: a study of the hemodynamics under variable modeling choices}

The mathematical and numerically sound framework developed in the previous sections is now applied in order to assess the influence of various modeling choices on the velocity and wall shear stress fields. We focus the study on the impact of setting boundary conditions, both at the inflow and the outflow, and on the rheological model for blood. Other possible sources of uncertainties, as for instance geometrical factors are briefly discussed in Section 5. For the sake of clarity and coherence, the numerical data reported in the sequel are presented in appropriate SI units.

\subsection{Influence of the outflow boundary condition}

The aim of this section is twofold: first, compare the solution obtained using a traction-free condition for the outflow boundaries with the solution of the coupled 3D-0D model; next, refine the analysis of the influence of the 3D-0D coupling on the solution, for different values of the parameters featuring the three-elements Windkessel model.

We start from the classical approximation assuming that for a Poisseuille flow, in a cylindrical vessel with length $l$ of constant circular section of radius $r_{0}$, the resistance and compliance can be estimated respectively by:

$$
R=\frac{8 \mu l}{\pi r_{0}^{4}}
$$

and

$$
C_{d, l}=\frac{3 \pi r_{0}^{3} l}{2 E h_{0}}
$$

where $h_{0}$ is the wall thickness and $E$ is Young modulus of the elastic vessel wall. In our computations, we set the proximal resistance $R_{p, l}$ to the following value indirectly derived from measurements for the internal jugular vein in supine position [7]:

$$
R_{p, l}=0.13 \pm 0.07 \times 10^{-3} \mathrm{mmHg} \cdot \mathrm{min} \cdot \mathrm{cm}^{-3}
$$

and then derive $R_{d, l}$ (the distal resistance) and $C_{d, l}$ (the distal compliance) accordingly. More precisely, we take $E=$ $0.5 \times 10^{6} \mathrm{~Pa}$ (Young modulus for the cerebral venous tree from [14]), $h_{0}$ as being $25 \%$ of the radius (acceptable approximation for cerebral vessels, according to [1]), and compute approximated values for the radius of the right and left internal jugular veins (denoted RIJV and LIJV in the sequel), using the formula $r_{0}=\sqrt{A_{0} / \pi}$, where $A_{0}$ is the area of the vessel. The values obtained are $r_{0}(R I J V)=2.59 \times 10^{-3} \mathrm{~m}$ and $r_{0}(L I J V)=2.34 \times 10^{-3} \mathrm{~m}$; we summarize the remaining parameters in Table 2 and Table 3, the entries of the first column of Table 2 being inferred from the range of values given in (22) for $R_{p, l}$. The last column in both tables is used to label in an explicit manner the three sets of parameters (corresponding to each raw) that will be subsequently used in the 3D-0D simulations. To these labels, we add the notation 3DFREE for the model using a traction-free condition at the outlet.

\begin{tabular}{rrrr}
\hline \multicolumn{1}{c}{$R_{p, l}$} & \multicolumn{1}{c}{$R_{p, l}$} & \multicolumn{1}{c}{$R_{d, l}$} & \multirow{2}{*}{ LABEL } \\
\cline { 1 - 3 } $\mathrm{mmHg} \cdot \mathrm{min} \cdot \mathrm{cm}^{-3}$ & $\mathrm{~Pa} \cdot \mathrm{s} \cdot \mathrm{m}^{-3}$ & $\mathrm{~Pa} \cdot \mathrm{s} \cdot \mathrm{m}^{-3}$ & \\
\hline $0.13 \times 10^{-3}$ & $1.0399116 \times 10^{6}$ & $1.0399116 \times 10^{8}$ & 3DW1 \\
$0.20 \times 10^{-3}$ & $1.59986 \times 10^{6}$ & $1.59986 \times 10^{8}$ & 3DW2 \\
$0.06 \times 10^{-3}$ & $0.479959 \times 10^{6}$ & $0.479959 \times 10^{8}$ & 3DW3 \\
\hline
\end{tabular}

Table 2: Resistances values in the three-element Windkessel model.

We first show in Figure 7 a comparison between the solution obtained when coupling the three-dimensional simulation with a lumped-parameter model, and the solution obtained with a standard traction-free outflow condition. The parameters used in the three-element Windkessel model correspond to the first raw of Tables 2 and 3 . Dissimilarities are more noticeable at the beginning of the simulation, but the metrics tend to stabilize afterwards, towards relatively small values for the velocity and slightly higher for the wall shear stress.

The impact of the parameters involved in the three-element Windkessel model is illustrated in Figure 8 . At the beginning of the simulation, the metrics reach a small peak value, but quite rapidly both indicators decrease and are stabilized around an even smaller value. Therefore, the analysis carried out in the present case shows that using a coupled 3D-0D model might 


\begin{tabular}{rrr}
\hline$C_{d, l}(\mathrm{RIJV})$ & $C_{d, l}(\mathrm{LIJV})$ & \multirow{2}{*}{ LABEL } \\
\cline { 1 - 2 } $\mathrm{m}^{3} \cdot \mathrm{Pa}^{-1}$ & $\mathrm{~m}^{3} \cdot \mathrm{Pa}^{-1}$ & \\
\hline $13.6534 \times 10^{-11}$ & $6.54286 \times 10^{-11}$ & 3DW 1 \\
$21.0052 \times 10^{-11}$ & $10.0659 \times 10^{-11}$ & 3DW2 \\
$6.30157 \times 10^{-11}$ & $3.01978 \times 10^{-11}$ & 3DW3 \\
\hline
\end{tabular}

Table 3: Compliance values in the three-element Windkessel model.

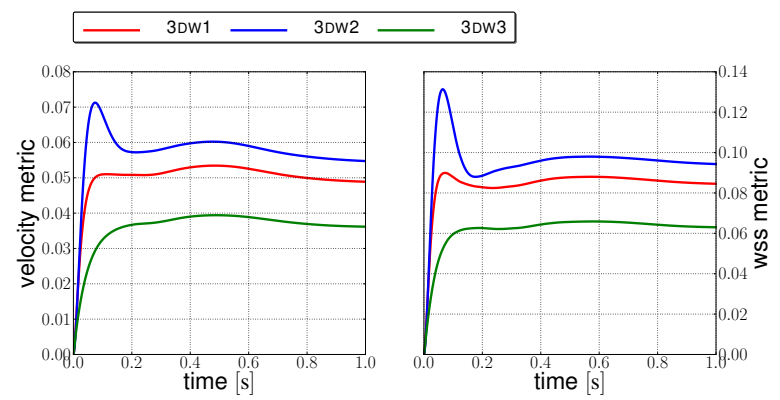

Figure 7: Effects of the outlet boundary condition: traction-free vs. 3D-0D coupling

be significant when a detailed analysis of the wall shear stress is relevant, but the choice of parameters is less influential, at least whilst kept in a physiological range described in the literature.
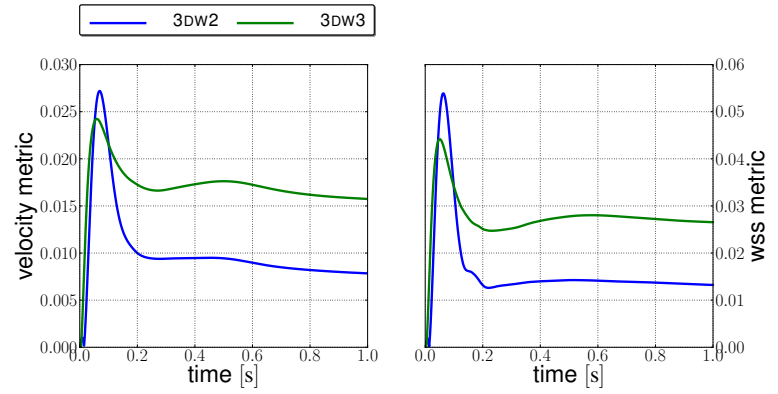

Figure 8: Influence of parameters.

In order to compare the results of the present simulations with some referenced data in the clinical literature, we extend the analysis to the values of flow rates and mean velocities at the level of the two outlets, corresponding to the left and right jugular veins, respectively. The results are displayed in Figure 9 In the left panel, we first focus on the results corresponding to the 3DFREE model: we checked that the sum of the flow rates corresponding to the represented outlets is the same as the flow at the inlet, thus ensuring mass conservation. The same property is respected when the 3D-0D models 3DW1, 3Dw2 and 3DW3 are used, but flow rates tend to balance in these simulations, with the best fit in the case of the highest resistance 3Dw2, as expected. The right panel illustrates the evolution of the mean velocity, with the most relevant unbalance between the two jugulars in the case of the 3DFREE model; the values tend to equilibrate when using a 3D-0D model, especially in the case 3Dw2, when the outflow resistance is the highest. All the obtained values are in the physiological range described in the literature, but since available data sets are not very rich and quite heterogeneous, further acquisitions and comparisons are needed in order to have more insightful information. 


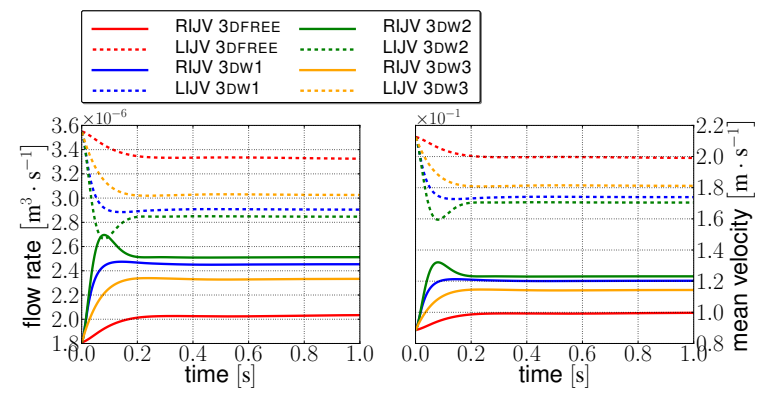

Figure 9: Flow rate and mean velocity measured at each outlet $\left(v_{\text {in }}=30 \times 10^{-3} \mathrm{~m} \cdot \mathrm{s}^{-1}\right)$

\subsection{Non-Newtonian characteristics of blood flow}

In this section, we aim at studying the effect on the hemodynamical quantities when using two different Generalized nonNewtonian models, in comparison with the classical Newtonian one. The parameters involved in the description of these models are the viscosities at zero and infinite shear rate [12, Chap. 6]:

$$
\begin{aligned}
\mu_{0} & =\lim _{\dot{\gamma} \rightarrow 0} \mu(\dot{\gamma})=56 \times 10^{-3} \mathrm{~Pa} \cdot \mathrm{s}, \\
\mu_{\infty} & =\lim _{\dot{\gamma} \rightarrow \infty} \mu(\dot{\gamma})=3.45 \times 10^{-3} \mathrm{~Pa} \cdot \mathrm{s},
\end{aligned}
$$

supplemented with material constants depending on the model, that correspond for the Carreau model to the values

$$
\lambda=3.313 \mathrm{~s}, \quad a=2, \quad n=0.3568,
$$

and for the Carreau-Yasuda model to the values

$$
\lambda=1.902 \mathrm{~s}, \quad a=1.25, \quad n=0.22,
$$

respectively.

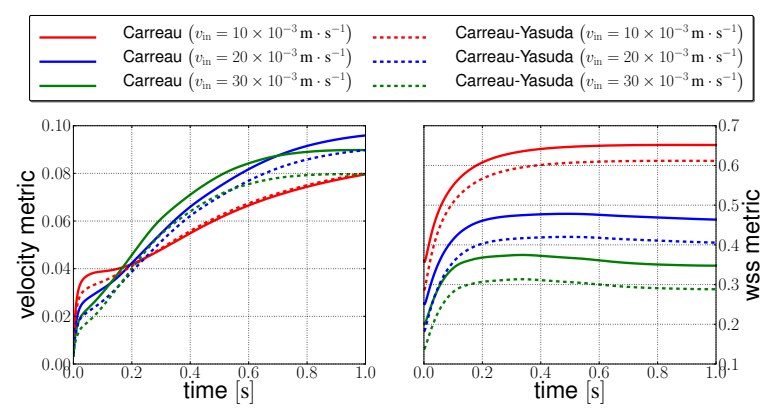

Figure 10: Effects of the generalized Newtonian Carreau and Carreau-Yasuda model for several input velocities. The reference solution for each curve is the Navier-Stokes Newtonian model, with the same inlet velocity.

Influence of the constitutive model for blood is showed in Figure 10 We compare the solution of three Newtonian simulations $\left(v_{\text {in }} \in\left\{10 \times 10^{-3}, 20 \times 10^{-3}, 30 \times 10^{-3}\right\} \mathrm{m} \cdot \mathrm{s}^{-1}\right)$ with non-Newtonian models using the same inlet velocity. First, there are relevant differences between the results of the non-Newtonian model, compared to the values obtained from 3D-0D model, in particular for wall shear stresses. According to the employed metrics, the use of a variable viscosity in the blood model has an important effect on the numerical predictions. There is a similar behavior between Carreau and Carreau-Yasuda models for both metrics, with a comparable impact (slightly less important for Carreau-Yasuda). Then, with respect to the velocity metric, we can see that the influence obtained from Carreau and Carreau-Yasuda models is similar, independently of the inlet velocity imposed. However, this is not the case with wall shear stress metric, where 
the influence of the non-Newtonian model changes significantly with the variability of the inlet velocity. We conclude that models for blood can have an important impact, particularly on the measure of wall shear stresses and directly related to the inlet boundary condition.

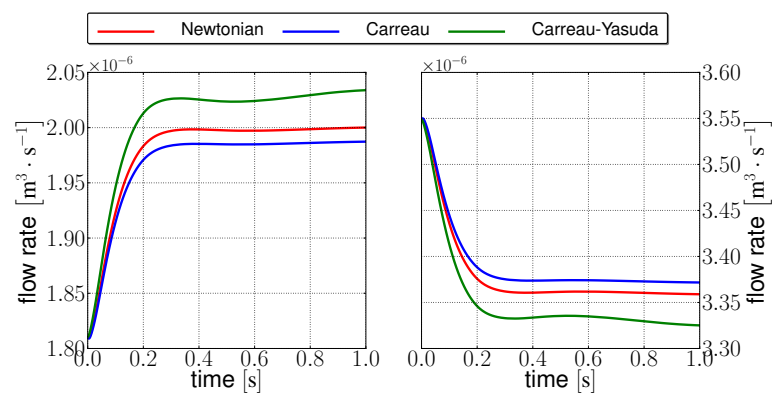

Figure 11: Flow rate measured at RIJV outlet (left) and LIJV outlet (right). Each result has been obtained with $v_{\text {in }}=$ $30 \times 10^{-3} \mathrm{~m} \cdot \mathrm{s}^{-1}$ and free-traction outflow condition.

Another comparison between the Newtonian and the non-Newtonian model is showed in Figure 11, displaying the flow rate measured at each outlet for several configurations. We can notice small differences between each model, compared to the previous analysis, see Section 4.1. However, Carreau-Yasuda model seems to have a flow rate with a more important evolution, compared to the other ones, that remain quasi-constant.

In order to localize the influence of the non-Newtonian model, we have exported fields associated to the metric velocity $S_{\mathbf{u}_{R}}\left(\mathbf{u}_{C}\right)$ and wall shear stress metric $T_{\tau_{R}}\left(\tau_{C}\right)$. Figures 12 and 13 illustrate an example of these visualization fields, in the case of the Carreau model. For the velocity metric, we see that major high sensitivity zones are localized mainly at bifurcations and recirculation zones. For the wall shear stress, the dissimilarities are slightly more global, but maximum quantities stay local and at same place than the velocity metric. It should be noted that, in order to visualize the velocity metric (see Figure 12), we had to remove a layer of tetrahedra close to the boundary because the metric has null values on the surface. Consequently, the geometry is not smooth anymore and some discontinuities appear in the small branches of the venous network.

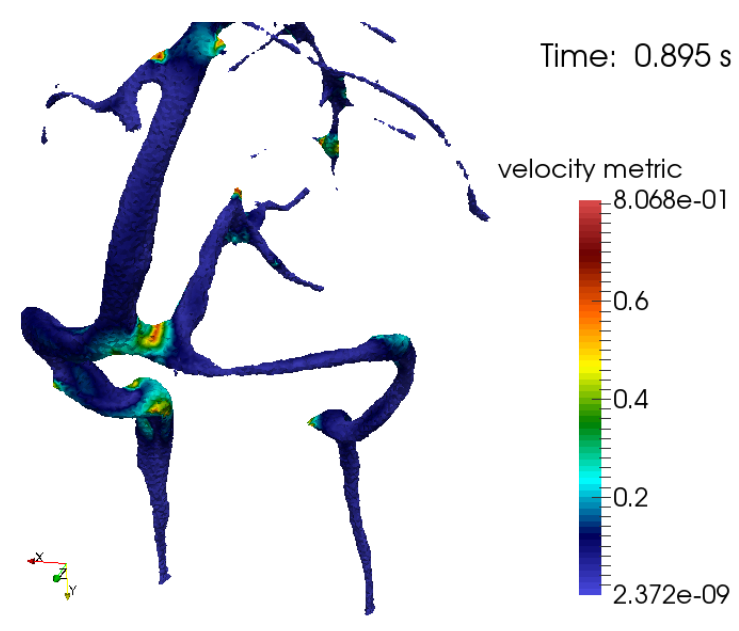

Figure 12: Visualization of the velocity metric (Carreau model $v_{\text {in }}=30 \times 10^{-3} \mathrm{~m} \cdot \mathrm{s}^{-1}$ ); only elements with threshold greater than $10^{-15}$ are plotted.

Finally, we present two visualizations of the viscosity field computed with the Carreau model. We start with Figure 14 obtained with same threshold geometry we previously described in the case of the velocity metric. This screenshot highlights a localization relation between the maximum values of viscosity and the velocity metric. Figure 15 also shows the viscosity field, but represented on the boundary surface. Furthermore, we have applied a threshold value in the scale visualization, in order to get a better illustration of the viscosity variability with respect to the wall shear stress metric. 


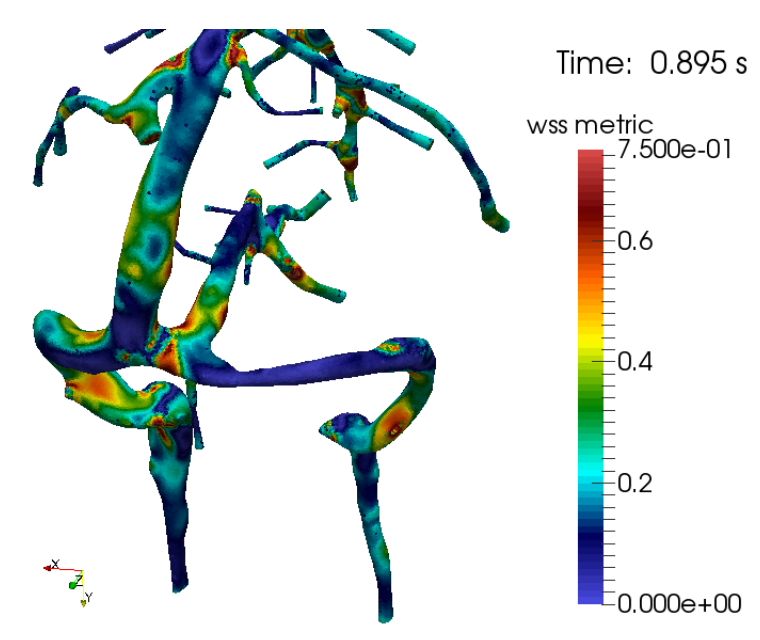

Figure 13: Visualization of the wall shear stress metric (Carreau model $v_{\text {in }}=30 \times 10^{-3} \mathrm{~m} \cdot \mathrm{s}^{-1}$ ).

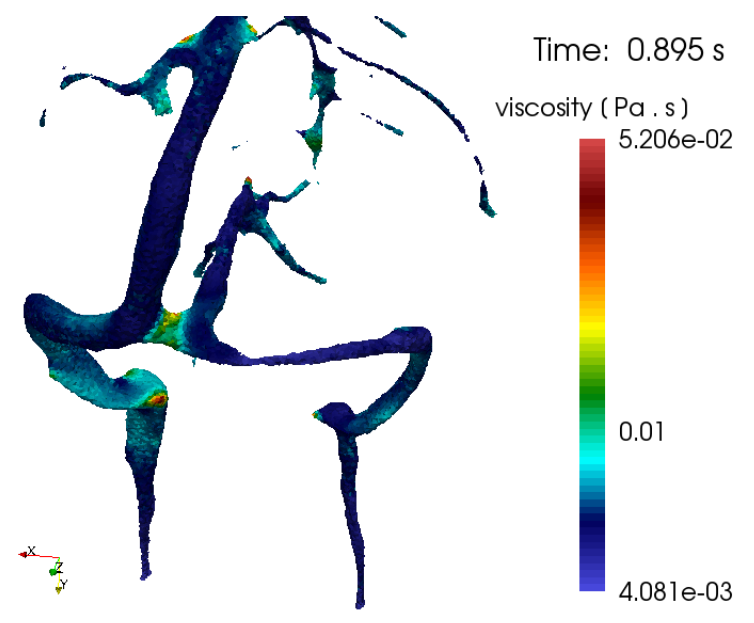

Figure 14: Visualization of the viscosity (Carreau model $v_{\text {in }}=30 \times 10^{-3} \mathrm{~m} \cdot \mathrm{s}^{-1}$ ).

\subsection{Influence of the inflow boundary condition}

We now present a sensitivity analysis with respect to the inlet velocity magnitude imposed at each inflow. The simulations were performed for several models (Newtonian 3D, non-Newtonian 3D and Newtonian 3D-0D). In each case, we take the reference solution with an inlet velocity magnitude equal to $10 \times 10^{-3} \mathrm{~m} \cdot \mathrm{s}^{-1}$. Then, we compute the influence of each model by taking $v_{i n} \in\left\{20 \times 10^{-3}\right.$ and $\left.30 \times 10^{-3}\right\} \mathrm{m} \cdot \mathrm{s}^{-1}$.

The results are displayed in Figure 16, where a grid refinement study is reported, and in Figure 17, where different models are compared. Both metrics are persistently higher in comparison with the previous cases analyzed in Sections 4.2 and 4.1, with a significant non-linear increase in the case of the wall shear stress metric. This should be compared to a Poisseuille approximation, frequently used in blood flow modeling, that predicts a linear increase of the wall shear stress when the velocity increases linearly. In the present case, where blood flow dynamics is complex and the geometry is also very complicated, this approximation is no more valid. It can also be noted that Newtonian 3D and Newtonian 3D-0D models present the same sensitivity for both velocity and wall shear stress metric; the same behavior can be found when comparing the two non-Newtonian models. Moreover, we can see in these figures that the non-Newtonian models have less influence compared to the Newtonian models. This effect is more significant in wall shear stress metric, but it is also visible in the velocity metric.

Finally, we plot the flow rate and the mean velocity measured at each outlet for several inlet velocities. The goal of this study is to show the impact of inlet velocity magnitude at the level of the jugular, where some clinical measures 


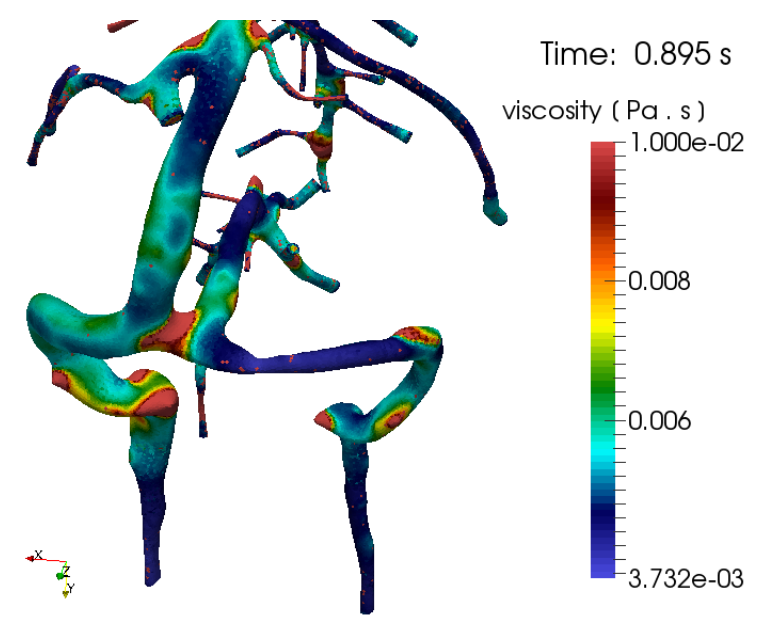

Figure 15: Visualization of the viscosity (Carreau model $v_{\text {in }}=30 \times 10^{-3} \mathrm{~m} \cdot \mathrm{s}^{-1}$ ) with threshold color $1 \times 10^{-2}$.
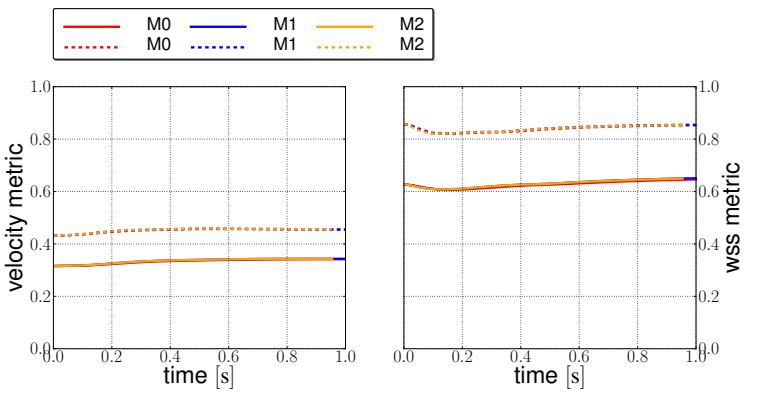

Figure 16: Effects of inlet velocity magnitude $v_{\text {in }}$. The reference solution for each level mesh is computed using $v_{\text {in }}=$ $10 \times 10^{-3} \mathrm{~m} \cdot \mathrm{s}^{-1}$. Continuous lines correspond to $v_{\text {in }}=20 \times 10^{-3} \mathrm{~m} \cdot \mathrm{s}^{-1}$ and dashed lines to $v_{\text {in }}=30 \times 10^{-3} \mathrm{~m} \cdot \mathrm{s}^{-1}$.

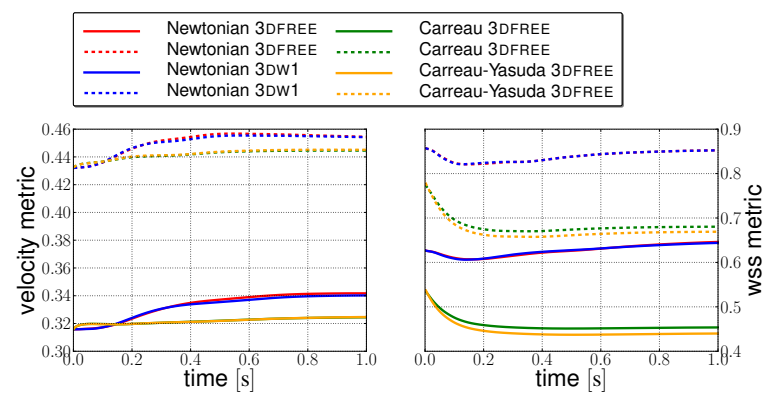

Figure 17: Effects of inlet velocity magnitude $v_{\text {in }}$. The reference solution for each model is computed by using $v_{\text {in }}=$ $10 \times 10^{-3} \mathrm{~m} \cdot \mathrm{s}^{-1}$. Continuous lines correspond to $v_{\text {in }}=20 \times 10^{-3} \mathrm{~m} \cdot \mathrm{s}^{-1}$ and dashed lines to $v_{\text {in }}=30 \times 10^{-3} \mathrm{~m} \cdot \mathrm{s}^{-1}$.

are available in the literature and subsequently infer a reasonable range of values for the imposed inlet velocity. A first report can be found in Figure 18. The simulations were performed with a Newtonian 3D model for three different input velocities. We notice that the RIJV outlet has a lower flow rate than the LIJV. The range of the mean velocity is $\left\{0.7 \times 10^{-1}\right.$ to $\left.2 \times 10^{-1}\right\} \mathrm{m} \cdot \mathrm{s}^{-1}$ (respectively $\left\{0.4 \times 10^{-1}\right.$ to $\left.1 \times 10^{-1}\right\} \mathrm{m} \cdot \mathrm{s}^{-1}$ for the LIJV (respectively RIJV), which are in the physiological range description in the clinical literature (see Section 11), with the closest values obtained for an inflow velocity $v_{\text {in }}=30 \times 10^{-3} \mathrm{~m} \cdot \mathrm{s}^{-1}$. Figure 19 reports similar comparisons, in the case of a 3D-0D Newtonian model. The interest of the coupled model is that, as already noticed in Section 4.1, in this case we recover a closer balance between both outlets in terms of the flow rate and mean velocity. 


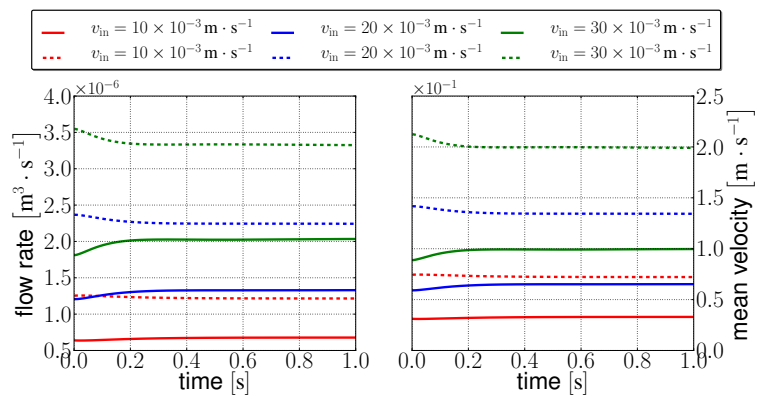

Figure 18: Flow rate and mean velocity measured at each outlet for several inlet velocities. Each result has been obtained with a Newtonian model 3DFREE. Continuous lines correspond to RIJV outlet and dashed lines to LIJV outlet.

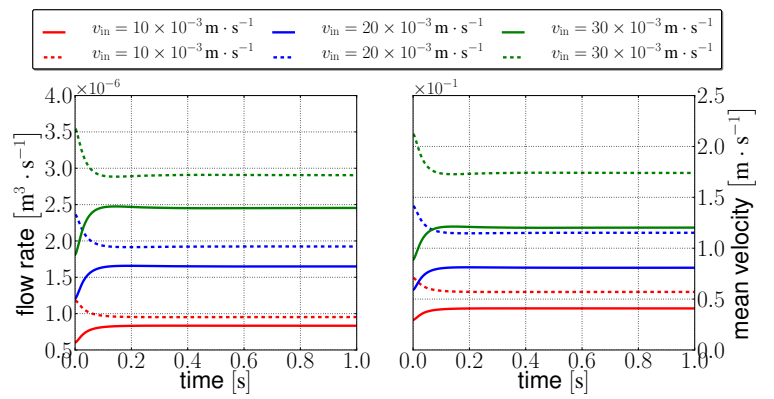

Figure 19: Flow rate and mean velocity measured at each outlet. Each result has been obtained with a Newtonian model 3D-0D. Continuous lines correspond to RIJV outlet and dashed lines to LIJV outlet.

\section{Conclusions and future directions}

The objective of the present work was to construct a sound mathematical, numerical and computational framework relevant to hemodynamic simulations and to assess within this context the effect of different modeling assumptions on cerebral venous blood flow dynamics at a macroscopic scale.

In order to guarantee reliable predictions, we first investigated the impact of the numerical strategy, via methodical grid refinement studies, variable initial condition implementations and different discretization techniques. The current methodology should be further developed, in particular by (i) improving linear solvers robustness and flexibility in the choice of temporal and spatial discretization methods; (ii) extending the present 3D - 0D model to a more complex multiscale framework [12, Chap. 11]; (iii) devising new strategies to compare solutions (as for instance statistical methods in the spirit of [11]).

The results of the present study showed that for cerebral veins blood flow modeling, the impact of setting the inlet boundary condition on the forces created by blood flow, is likely greater than for other modeling assumptions. Therefore, they highlighted the importance of deriving values for these conditions from clinically measured data at some probe locations, in order to enhance the accuracy of the computed hemodynamical quantities of interest. It was also found that in the present case, the impact of the rheological model used for blood is important, with a strong emphasis on the computation of the wall shear stresses. The overview of the results on the whole geometry, when using a Generalized non-Newtonian model, shows that significant differences are localized especially at zones of maximum viscosity. In view of these findings, other more complex models should be implemented for comparison, for instance Casson model incorporating the hematocrit dependence [11]. A reduced impact was found regarding the outflow treatment: the 3D - 0D coupling has the advantage of equilibrating flow rates and mean velocities at the level of the jugulars, thus allowing to obtain physiological values; however the influence of the parameters in the three-element Windkessel model was not very significant in the range described in the clinical literature. Even so, because of the heterogeneous individual venous flow and variable side dominance [23], and also because of the possible modifications in local resistance and compliance values in pathological cases, a larger range of values should be tested. Furthermore, work to include the influence of the geometrical approximation is needed, as well as the study of the impact of the gravity force, which plays a major role in the cerebral venous drainage [13]. 
The present work should also be seen as an important step in the construction of a computational model for the whole cerebral circulation, after coupling with the arterial network and the capillary bed.

\section{Acknowledgments}

The authors acknowledge PRACE for awarding access to resource SuperMUC based in Germany at LRZ, the French National Research Agency (ANR) for the funding of the project Vivabrain (Grant Agreement ANR-12-MONU-0010), the support of the Center for Modeling and Simulation in Strasbourg and the Tier-2 computing centers in Strasbourg and Grenoble.

\section{References}

\section{References}

[1] J. Alastruey, K.H. Parker, S.M. Byrd, J. Peiro, S.J. Sherwin. Modelling the circle of Willis to assess the effects of anatomical variations and occlusions on cerebral flows. J. Biomech., Vol. 40 1794-1805, 2007.

[2] C. Bertoglio, A. Caiazzo, M.A. Fernández. Fractional-step schemes for the coupling of distributed and lumped models in hemodynamics. SIAM J. Sci. Comp., Vol. 35, 551-575, 2013.

[3] A. Caiazzo, G. Montecinos, L.O. Müller, E.M. Haacke, E.F. Toro. Computational haemodynamics in stenotic internal jugular veins J. Math. Biol., in press.

[4] C. Caldini Queiros, V. Chabannes, M. Ismail, G. Pena, C. Prud'Homme, M. Szopos, R. Tarabay. Towards large-scale three-dimensional blood flow simulations in realistic geometries., ESAIM Proc., Vol. 43, 195-212, 2013.

[5] V. Chabannes. Vers la simulation numérique des écoulements sanguins. Phd Thesis, Université de Grenoble, 2013.

[6] V. Chabannes, G. Pena, and C. Prud'Homme. High-order fluid-structure interaction in 2D and 3D application to blood flow in arteries. Journal of Computational and Applied Mathematics, Vol. 246, 1-9, 2013.

[7] S. Cirovic, C. Walsh, W.D. Fraser, A. Gulino. The effect of posture and positive pressure breathing on the hemodynamics of the internal jugular vein. Aviat. Space Environ. Med., Vol. 74, 125-131, 2003.

[8] V. Doyeux, V. Chabannes, C. Prud'Homme, M. Ismail. Simulation of vesicle using level set method solved by high order finite element. ESAIM: Proceedings, Vol. 38, 335-347, 2012.

[9] V. Doyeux, Y. Guyot, V. Chabannes, C. Prud'homme, M. Ismail. Simulation of two-fluid flows using a finite element/level set method. Application to bubbles and vesicle dynamics. Journal of Computational and Applied Mathematics, Vol. 246, 251-259, 2013.

[10] Ø. Evju. Sensitivity Analysis of Simulated Blood Flow in Cerebral Aneurysms. Master's Thesis. DUO, University of Oslo, 2011.

[11] Ø. Evju, K. Valen-Sendstad, K.-A. Mardal. A study of wall shear stress in 12 aneurysms with respect to different viscosity models and flow conditions. J. Biomech., Vol. 46, 2802-2808, 2013.

[12] L. Formaggia, A. Quarteroni, A. Veneziani, Cardiovascular mathematics, MS\&A. Modeling, Simulation and Applications, Vol. 1, Springer-Verlag Italia, Milan, 2009.

[13] J. Gisolf, J.J. van Lieshout, K. van Heusden, F. Pott, W.J. Stok, J.M. Karemaker. Human cerebral venous outflow pathway depends on posture and central venous pressure. J. Physiol. Vol. 560, 317-327, 2004.

[14] H. Ho, K. Mithraratne, P. Hunter. Numerical Simulation of Blood Flow in an Anatomically-Accurate Cerebral Venous Tree, IEEE Trans. Med. Imag., Vol. 32, 85-91, 2013.

[15] O. Miraucourt, S. Salmon, M. Szopos, M. Thiriet. Blood flow simulations in the cerebral venous network. 3rd International Conference on Computational and Mathematical Biomedical Engineering (CMBE 2013) Proc., 187-190, 2013. 
[16] O. Miraucourt, O. Génevaux, M. Szopos, M. Thiriet, H. Talbot, S. Salmon, N. Passat. 3D CFD in complex vascular systems: A case study. International Symposium on Biomedical Simulation (ISBMS), Lecture Notes in Computer Science, Vol. 8789, 86-94, Springer, 2014.

[17] L.O. Müller, E.F. Toro. A global multiscale mathematical model for the human circulation with emphasis on the venous system, Int. J. Numer. Meth. Biomed. Engng., Vol. 30, 681-725, 2014.

[18] S. Ogoh, K. Sato, J.P. Fisher, T. Seifert, M. Overgaard, N.H. Secher. The effect of phenylephrine on arterial and venous cerebral blood flow in healthy subjects. Clinical Physiology and Functional Imaging Vol. 31, 445-451, 2011.

[19] C. Prudhomme, V. Chabannes, V. Doyeux, M. Ismail, A. Samake, G. Pena. Feel++: A Computational Framework for Galerkin Methods and Advanced Numerical Methods. http: //www. feelpp.org, ESAIM Proc., Volume 38, 429-455, 2012.

[20] A. Quarteroni, Numerical models for differential problems, MS\&A. Modeling, Simulation and Applications, Vol. 2, Springer-Verlag Italia, Milan, 2009.

[21] B. Schaller. Physiology of cerebral venous blood flow: From experimental data in animals to normal function in humans. Brain Res. Rev., Volume 46, 243-260, 2004.

[22] D.M. Sforza, R. Löhner, C. Putman, J.R. Cebral. Hemodynamic analysis of intracranial aneurysms with moving parent arteries: Basilar tip aneurysms. Int. J. Numer. Meth. Biomed. Engng., Vol. 26, 1219-1227, 2010.

[23] S. Stoquart-Elsankari, P. Lehmann, A. Villette, M. Czosnyka, M.E. Meyer, H. Deramond, O. Balédent. A phasecontrast MRI study of physiologic cerebral venous flow. J. Cerebral Blood Flow and Metabolism, Vol. 29, 1208-1215, 2009.

[24] K. Valen-Sendstad, D.A. Steinman. Mind the gap: Impact of Computational Fluid Dynamics solution strategy on prediction of intracranial aneurysm hemodynamics and rupture status indicators. Am. J. Neuroradiol., Vol. 35, 536$543,2014$. 